\title{
ARTICLE
}

\section{Results of hysterectomy for persistent cervical cancer after complete radiotherapy in Srinagarind Hospital}

Author

Bandit Chumworathayi, MD, PhD, Suttiwan Thongyot, MD, Sanguanchoke Luanratanakorn, MD, Banchong Udomthavornsuk, MD

\section{Affiliation}

Division of Gynecologic Oncology, Department of Obstetrics and Gynecology, Faculty of Medicine, Khon Kaen University, Khon Kaen, 40002, THAILAND

\section{Correspondence}

Bandit Chumworathayi

Associate Professor, Division of Gynecologic Oncology,

Department of Obstetrics and Gynecology, Faculty of Medicine,

Khon Kaen University, Khon Kaen, 40002, THAILAND

Email: bchumworathayi@gmail.com

\section{$\underline{\text { Abstract }}$}

Objectives: To analyze the survival outcomes and to determine the surgical complications of patients with persistent cervical cancer who had undergone hysterectomy after completion of radiotherapy at Srinagarind Hospital, Khon Kaen University, Thailand between January 1996 and December 2010.

Methods: The medical records of patients who met the study criteria were reviewed. The inclusion criteria are all patients who have residual cervical cancer after complete radiation therapy and biopsy proven. Extracted data included patient's age, stage, histological subtype, type of radiotherapy received, type of hysterectomy procedure, pathological results, surgical complications, and survival.

Results: Forty-three women suitable for study inclusion were identified. The majority of women $(53.5 \%)$ were in stage IIB. Thirteen $(30.2 \%)$ women received concurrent chemoradiation while the remaining $30(69.8 \%)$ underwent radiotherapy alone. Thirty-four $(79.1 \%)$ women underwent extrafascial hysterectomy (EH) while the remaining 9 (20.9\%) underwent radical hysterectomy $(\mathrm{RH})$. Median operative time and median amount of estimated blood loss were higher among women undergoing RH compared to those who underwent EH. Overall, grade III-IV complications were noted in $34.9 \%$ of patients. The rate of grade III-IV complications was higher among women undergoing $\mathrm{RH}$ compared to those in $\mathrm{EH}$ group $(44.4 \%$ vs. $32.4 \%$, respectively). The 5-year survival rate of all women was 54.6\%. Median overall survival of all patients was 6.25 years. Median overall survival of women undergoing RH was comparable to those who underwent EH (6.25 years vs. 6.84 years, respectively).

Conclusion: Hysterectomy in women who were initially treated with curative intent radiotherapy but subsequently found to have residual tumor may improve survival outcomes, allowing a 5year survival rate of approximately 55\%. The incidence of severe complication was considerably high particularly among those women who had undergone radical hysterectomy.

Keywords: Hysterectomy, cervical cancer, radiotherapy, residual lesion, persistent disease 


\section{Introduction}

Worldwide, as well as Thailand, cervical cancer is the second most common after breast cancer among female cancers. The age standardized incident rate (ASR) of cervical cancer and deaths from this cancer in Thailand is 24.5 and 12.8 per 100,000 women per year, respectively. In Thailand, there were estimated 10,000 newly diagnosed cervical cancer cases and 5,000 deaths from this disease in 2008. In December, 2018, Thai Ministry of Public Health has reported the number of 9,000 annual newly diagnosed cervical cancer cases and 5,000 deaths. These numbers were still persistently high. ${ }^{(1)}$ Thus, effective screening and treatment of this malignant disease are mandatory.

Radiotherapy has been acknowledged as the principal treatment of cervical cancer. The single most important advantage of radiotherapy is that it can be applied in all cases of cervical cancer of any clinical stages. In previous randomized controlled trial, women with early stages of cervical cancer undergoing radiotherapy carried similar survival outcomes when compared to those who underwent standard radical surgery. So, decision making of the optimum therapy for each patient with early stages cervical cancer should be taken in to account of other important clinical variables including menopausal status, age, medical illness, patients' preference, and the competency of surgical team. ${ }^{(2)}$ However, radiotherapy becomes the only viable treatment option in patients with advanced stage of this cancer.

Although the efficacy of radiotherapy for treating cervical cancer has improved after an incorporation of the chemotherapeutic agent into the traditional radiotherapy protocol, or the so-called concurrent chemoradiation ${ }^{(3)}$, there are certain numbers of patients however suffer from post-radiotherapy residual disease particularly in those with bulky lesion or in advanced stage of disease. ${ }^{(4-7)}$ The presence of residual lesion after completion of curative intent radiotherapy or chemoradiation poses a negative impact to patients' survival. Thus, tailored interventions such as adjuvant hysterectomy might be considered in order to reduce the risk of disease progression.

The aim of this study was to analyze the survival outcomes and to determine the surgical complications of patients with cervical cancer who had undergone hysterectomy after completion of radiotherapy for curative intent intervention at Srinagarind Hospital, Khon Kaen University, Thailand.

\section{Materials and methods}

After approval of the Research Ethics Committee, the medical records of patients with cervical cancer who had undergone hysterectomy for persistent cervical cancer after 
completion of radiotherapy between January 1996 and December 2010 at Department of Obstetrics and Gynecology, Srinagarind Hospital, Faculty of Medicine, Khon Kaen University, Thailand, were reviewed. Inclusion criteria are all women who have residual tumor after complete radiotherapy and had biopsy for pathological proven before surgery. Exclusion criteria are others women who did not have biopsy proven for residual tumor before surgery or having progressive disease.

After completion of radiotherapy, patients were followed up by pelvic examination and Pap smear every 3 months in first year, every 4 months in second year, every 6 months in $3^{\text {rd }}-5^{\text {th }}$ year, and then once a year for whole life. All of the patients recruited in this study had abnormal Pap smear during their first or second follow-up after completion of radiotherapy, leading them to biopsy proven of residual tumor. Radiotherapy or surgical technique did not change during this period. Only intervention changed was concurrent chemoradiation therapy (CCRT) beginning in 2004.

General practice for persistent cervical cancer in Srinagarind hospital are surgery if operable case, or palliative chemotherapy and best supportive care in inoperable case. Extracted data included patient's age, clinical stage of cancer, histology subtype, type of radiotherapies (radiotherapy alone or concurrent chemoradiation), type of hysterectomy procedures (extrafascial hysterectomy or radical hysterectomy), clinical volume of residual lesion determined in maximal dimension during gross specimen examination in the operative room, intraoperative time and amount of blood loss, pathological results of hysterectomy specimens, surgical complications, and survival. Staging of cervical cancer were clinically assessed using the International Federation of Gynecologic and Obstetrics (FIGO) nomenclature. Surgical complications were classified according to the European Organization for Research and Treatment of Cancer (EORTC) grading system.

Data were analyzed using the STATA software. Descriptive data were present as number (percentage) or median (range) when appropriate. The survival outcome of interest in this study was overall survival (OS) which was defined as a time frame between the date of hysterectomy procedure carried out and the date of death from any causes. Survivors were censored at the time of last contact for analysis. Overall survival probabilities were estimated according to the Kaplan-Meier methods.

\section{Results}

During the study period, 43 women with cervical cancer who had undergone hysterectomy after completion of radiotherapy were identified. The median age was 46 years. 
Twenty-two (51.2\%) had squamous cell carcinoma histology while adenocarcinoma histology was noted in 20 (46.5\%) women. The only one patient is adenosquamous histologic subtype. The majority of women were clinically diagnosed with stage IIB $(23,53.5 \%)$, followed by stage IIIB $(10,23.3 \%)$, stage IB2 $(5,11.6 \%)$, stage IIA $(3,7.0 \%)$, and stage IB1 $(2,4.7 \%)$.
Thirteen (30.2\%) women received Concurrent chemoradiation as their primary therapies while the remaining $30 \quad(69.8 \%)$ underwent radiotherapy alone. Median time from completion of radiotherapy to the date of hysterectomy performed was 101 days with a range of 36 days to 223 days. Median of largest diameter of residual cervical lesion was $2 \mathrm{~cm}$.

Table 1 Baseline characteristics of the patients stratified by the types of hysterectomy procedure

\begin{tabular}{|c|c|c|c|}
\hline \multirow[t]{2}{*}{ Patients characteristics } & \multirow{2}{*}{$\begin{array}{l}\text { All patients } \\
\quad(\mathrm{N}=43)\end{array}$} & \multicolumn{2}{|c|}{ Types of procedure } \\
\hline & & $\begin{array}{c}\text { EH } \\
(\mathrm{N}=34)\end{array}$ & $\begin{array}{c}\mathbf{R H} \\
(\mathrm{N}=9)\end{array}$ \\
\hline Age (years) & $46(16-67)$ & $46(16-67)$ & $42(33-57)$ \\
\hline \multicolumn{4}{|l|}{ Histology } \\
\hline - Squamous cell carcinoma & 22 & 15 & 7 \\
\hline - Adenocarcinoma & 20 & 18 & 2 \\
\hline - Others & 1 & 1 & 0 \\
\hline \multicolumn{4}{|l|}{ FIGO stage } \\
\hline - IB1 & 2 & 1 & 1 \\
\hline$-\mathrm{IB} 2$ & 5 & 4 & 1 \\
\hline - IIA & 3 & 3 & 0 \\
\hline - IIB & 23 & 17 & 6 \\
\hline - IIIB & 10 & 9 & 1 \\
\hline \multicolumn{4}{|l|}{ Radiation type } \\
\hline - CCRT & 13 & 13 & 0 \\
\hline - Radiation alone & 30 & 21 & 9 \\
\hline Interval time (days)* & $101(36-223)$ & $103(36-223)$ & $78(51-190)$ \\
\hline Residual tumor (cm) & $2(0-4)$ & $1(0-3)$ & $1(0.5-4)$ \\
\hline Operative time (min) & $130(85-225)$ & $125(85-195)$ & $195(125-225)$ \\
\hline Estimated blood loss (ml) & $400(50-1300)$ & $300(50-800)$ & $800(500-1300)$ \\
\hline \multicolumn{4}{|l|}{ Pathological results } \\
\hline - free margins & 29 & 22 & 7 \\
\hline - closed to vaginal margin & 2 & 2 & 0 \\
\hline - closed to cervical serosa & 3 & 2 & 1 \\
\hline - positive margin & 8 & 7 & 1 \\
\hline - necrotic tumor & 1 & 1 & 0 \\
\hline
\end{tabular}

Abbreviation: EH, extrafascial hysterectomy; RH, radical hysterectomy; FIGO, International Federation of Gynecologic and Obstetrics; CCRT, concurrent chemoradiation

*From completion of radiation to surgery

Data were present as number or median (range) 
In this study, thirty-four $(79.1 \%)$ women underwent extrafascial hysterectomy (EH) while the remaining 9 (20.9\%) underwent radical hysterectomy $(\mathrm{RH})$. Table 1 displays the baseline characteristics of patients in this study cross-tabulated by the types of hysterectomy procedures. Median operative time and median amount of estimated blood loss during operation were higher among women undergoing RH compared to those who underwent $\mathrm{EH}$. Women undergoing $\mathrm{RH}$ were more likely to be reported as having free surgical margins determined by pathological examination than those who underwent $\mathrm{EH}$ (77.8 \% vs. $64.7 \%$, respectively). A woman who has residual disease of $0 \mathrm{~cm}$ is a woman who has abnormal cytology for follow up after complete radiation therapy and then she was send to colposcopic biopsy and then the pathological result are adenocarcinoma and the pathological result of hysterectomy did not show residual tumor.

The severe surgical complications (grade III-IV) were detailed in Table 2. Overall, grade III-IV complication was noted in $34.9 \%$ of patients. The three most common complication sites were surgical wound, followed by urinary bladder, and ureter and rectum. When stratified by type of procedures, grade III-IV complication rate was noted to be at $44.4 \%$ of women undergoing $\mathrm{RH}$ which was higher than those reported among women undergoing $\mathrm{EH}(32.4 \%)$.

Table 2 Surgical complications according to EORTC grading system

\begin{tabular}{lcccccc}
\hline \multirow{2}{*}{ Site } & \multicolumn{2}{c}{$\begin{array}{c}\text { All patients } \\
(\mathrm{N}=15 / 43)\end{array}$} & \multicolumn{2}{c}{ EH } & \multicolumn{2}{c}{ RH } \\
& \multicolumn{2}{c}{ (N=11/34) } & \multicolumn{2}{c}{$(\mathrm{N}=4 / 9)$} \\
\hline grade III & grade IV & grade III & grade IV & grade III & grade IV \\
\hline Rectum & - & 2 & - & 1 & - & 1 \\
Small bowel & - & 1 & - & 1 & - & - \\
Urinary bladder & 2 & 1 & 2 & - & - & 1 \\
Ureter & - & 2 & - & 1 & - & 1 \\
Wound & 4 & - & 4 & - & - & - \\
Lymphedema & 1 & - & 1 & - & - & - \\
Sepsis & 1 & 1 & 1 & - & - & 1 \\
\hline
\end{tabular}

Abbreviation: EH, extrafascial hysterectomy; RH, radical hysterectomy; EORTC, European Organization for Research and Treatment of Cancer

Data were present as number 
During the study period, only 3 patients died of the disease, others were found at their last follow-up. No patient lost to follow up. Patients' deaths were detected by household registration data of Thai government on the web. The probability of survival of all patients in this study was displayed in Figure 1. The 5year survival rate was $54.6 \%$ with a $95 \%$ confidence interval (CI) of $37.3 \%$ to $69.0 \%$. An estimated 10-year OS was $43.0 \%$ with a $95 \%$ CI of $23.9 \%$ to $60.8 \%$. Median OS of all patients was 6.25 years. When stratified by types of hysterectomy procedures, the median OS of women undergoing RH was comparable to those who underwent EH (6.25 years vs. 6.84 years, respectively).

Figure 1 Survival of all 43 patients

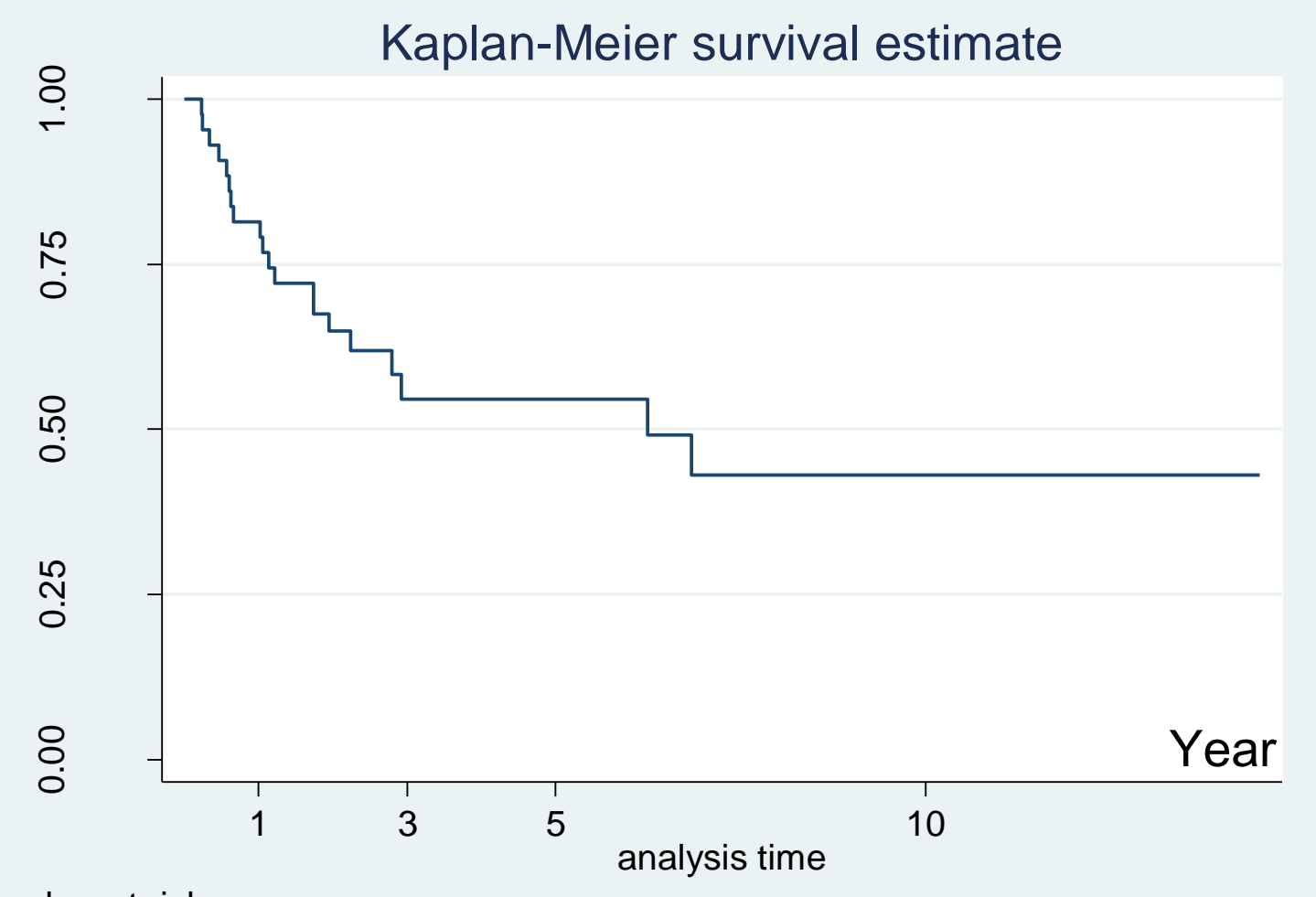

Number at risk

\section{Discussion}

Knowing that the presence of residual tumor after curative intent radiotherapy put the patients with cervical cancer at a higher risk of disease progression and cancer-related deaths, ${ }^{(4-}$
7) hysterectomy might have a place in the multimodality managements of such women. As for us, we noted that hysterectomy performed in women with various stages of cervical cancer who were initially treated with 
radiotherapy or concurrent chemoradiation but subsequently found to have residual tumor may improve the survival outcomes, allowing a 5year survival rate of approximately $55 \%$ and median overall survival of 6.25 years.

Role of routine adjuvant extrafascial hysterectomy for early-stage cervical cancer with bulky lesion has gradually gained attention owing to a higher risk of having residual lesion after radiotherapy. In 2003, the Gynecologic Oncology Group (GOG) published the randomized trial determining benefit of adjuvant extrafascial hysterectomy in bulky stage IB cervical cancer. Unfortunately, this trial was unable to demonstrate survival benefit with the use of routine extrafascial hysterectomy. ${ }^{(8)}$ Thus, routine adjuvant hysterectomy after radiotherapy in women with early-stage cervical cancer has not recommended in the current practice and should generally be reserved for meticulously selected patients who have residual tumor after completion of radiotherapy courses.

Intervals from RT to surgery in our study were varied from 36 to 223 day, because inclusion criteria for diagnosis of persistent disease were residual tumors after radiation therapy completed. Thus, some cases were early but some cases were delayed to diagnosed, depending upon judgment of their surgeons or radiotherapists.
Women with advanced stage of cervical cancer carried a higher risk of harboring residual tumor after radiotherapy than those in early-stage of disease. ${ }^{(4)}$ In our series, the majority of cervical cancer patients who were found to have residual disease were initially diagnosed with locally advanced stage. Nevertheless, there is limited published evidence regarding the role of hysterectomy after curative intent radiotherapy in women with advanced cervical cancer. In a retrospective, multicenter study by Motton et $\mathrm{al}^{(4)}$ which was conducted to determine the outcome of hysterectomy after concurrent chemoradiation in advanced cervical cancer, approximately $70 \%$ of cases were able to undergo complete hysterectomy. Interestingly, there was a trend to have a slightly longer disease-free survival at 48 months among women who underwent hysterectomy compared to those who did not. Thus, if it is rendered operable, hysterectomy can be considered as an alternative treatment in women with advanced cervical cancer who had residual lesion after completion of radiotherapy.

One of the major issues regarding hysterectomy after radiotherapy is the risk of severe perioperative complications. In our series, grade III-IV complication was noted in $34.9 \%$ of patients which was consistent with the previous reported incidence that ranged from $20 \%$ to $40 \% .^{(4,9,10)}$ The possible predictor 
for the risk of having severe complication in our series is the type of hysterectomy. Our study noted that women undergoing radical hysterectomy carried a higher rate of severe complication than those who underwent extrafascial hysterectomy $(44.4 \%$ versus $32.4 \%$, respectively) which reaffirmed the correlation between the radicality of hysterectomy procedure and rate of severe complication as has been reported previously. ${ }^{(4)}$ This vital information should be taken into account during patients counseling for treatment planning.

In our series, when comparing between two types of hysterectomy procedures, median OS of women undergoing RH was comparable to those who underwent EH (6.25 years vs. 6.84 years, respectively). This finding was in line with the results from the previous report which found that extended hysterectomy has not only failed to demonstrate any advantage in term of survival, but also posed a higher risk of severe surgical complications. ${ }^{(4)}$ Therefore, it might be logical to suggest extrafascial hysterectomy as a procedure of first choice. Radical hysterectomy should be reserved for women who are at the risk of having positive surgical margins owing to it could lower the rate of surgical margin involvement as noted in our study.

There are growing evidences highlighted the distinction of basic molecular pathogenesis between squamous cell carcinoma and adenocarcinoma of cervix. ${ }^{(11-14)}$ When considering particular studies derived from a large cohort or population-based, patients with cervical adenocarcinoma carried poorer response rate, used longer time to achieve complete response, and had inferior survival outcomes than that in patients with squamous cell carcinoma. ${ }^{(15-17)}$ For example, the recent report using the Surveillance, Epidemiology, and End Results (SEER) database which evaluated the results of 24,562 women with cervical cancer observed that adenocarcinoma histology negatively impacted survival for both early and advanced-stage cervical cancer. In addition, the negative impact on the survival of adenocarcinoma histology remained statistically significant even after performing sensitivity analyses to determine the influence of the use of concurrent chemoradiation. ${ }^{(18,19)}$ These findings emphasize the relatively insensitive of adenocarcinoma to the current standard treatment compared to squamous cell carcinoma. Unsurprisingly, approximately 50\% of women who found to have residual lesion after curative intent radiotherapy in our series had adenocarcinoma histology despite the fact that cervical adenocarcinoma generally accounts for only around $10 \%$ of all cervical cancer cases. Hence, the development of specific treatment strategy aimed to improve survival in patients with cervical 
adenocarcinoma is needed. Role of adjuvant hysterectomy for women with cervical adenocarcinoma also merits further investigation. ${ }^{(20)}$

We recognized a number of important limitations in our study. This series contained a small number of patients. As such, the comparisons of survival difference between the various important factors i.e. FIGO stage, types of surgical intervention, types of previous radiotherapy, were limited. Because of the retrospective study by nature, the uniform managements regarding the preoperative investigation and criteria for choosing the appropriate surgical procedure were therefore impossible. Finally, our series lacked data on timing and distribution of recurrence, thus it was unable to analyze disease-free survival.

In conclusion, hysterectomy in women who were initially treated with curative intent radiotherapy and subsequently found to have residual tumor may improve survival outcomes, allowing a 5-year survival rate of approximately 55\%. Grade III-IV complications were noted in $34.9 \%$ of patients. Women undergoing radical hysterectomy had a higher incidence of severe complication but carried a lower rate of surgical margin involvement compared to those who underwent extrafascial hysterectomy.

\section{Conflicts of interest}

None declared.

NOTE: This research manuscript, without any grant or funding source, was orally presented in the annual meeting of Thai Gynecologic Oncology Society (TGCS) in 2012. 


\section{References}

1. Thai Ministry of Public Health. Cervical cancer incidence rate in 2018. Available at https://hdcservice.moph.go.th/hdc/reports/repor t.php? source=formated/ncd.php\&cat_id=6a1fdf 282fd28180eed7d1cfe0155e11\&id=f3a9cabcd1 b83af2ad8d8db6d9b6735c Access date $6^{\text {th }}$ December, 2018.

2. Landoni F, Maneo A, Colombo A, Placa F, Milani R, Perego P, et al. Randomised study of radical surgery versus radiotherapy for stage $\mathrm{Ib}$ IIa cervical cancer. Lancet 1997; 350(9077):535-40.

3. Green J, Kirwan J, Tierney J, Vale C, Symonds P, Fresco L, et al. Concomitant chemotherapy and radiation therapy for cancer of the uterine cervix. Cochrane Database Syst Rev 2005:CD002225.

4. Motton S, Houvenaeghel G, Delannes M, Querleu D, Soule-Tholy M, Hoff J, et al. Results of surgery after concurrent chemoradiotherapy in advanced cervical cancer: comparison of extended hysterectomy and extrafascial hysterectomy. Int J Gynecol Cancer 2010;20(2):268-75.

5. Kunos C, Ali S, Abdul-Karim FW, Stehman FB, Waggoner S. Posttherapy residual disease associates with long-term survival after chemoradiation for bulky stage 1B cervical carcinoma: a Gynecologic Oncology Group study. Am J Obstet Gynecol 2010;203(4):351 e1-8.

6. Morice P, Uzan C, Zafrani Y, Delpech Y, Gouy S, Haie-Meder C. The role of surgery after chemoradiation therapy and brachytherapy for stage IB2/II cervical cancer. Gynecol Oncol 2007;107(1 Suppl 1):S122-4.

7. Ota T, Takeshima N, Tabata T, Hasumi K, Takizawa K. Adjuvant hysterectomy for treatment of residual disease in patients with cervical cancer treated with radiation therapy. Br J Cancer 2008;99(8):1216-20.

8. Keys HM, Bundy BN, Stehman FB, Okagaki T, Gallup DG, Burnett AF, et al. Radiation therapy with and without extrafascial hysterectomy for bulky stage IB cervical carcinoma: a randomized trial of the Gynecologic Oncology Group. Gynecol Oncol 2003;89(3):343-53.

9. Coleman RL, Keeney ED, Freedman RS, Burke TW, Eifel PJ, Rutledge FN. Radical hysterectomy for recurrent carcinoma of the uterine cervix after radiotherapy. Gynecol Oncol 1994;55(1):29-35. 
10. Azria E, Morice P, Haie-Meder C, Thoury A, Pautier P, Lhomme C, et al. Results of hysterectomy in patients with bulky residual disease at the end of chemoradiotherapy for stage IB2/II cervical carcinoma. Ann Surg Oncol 2005;12(4):332-7.

11. Contag SA, Gostout BS, Clayton AC, Dixon MH, McGovern RM, Calhoun ES. Comparison of gene expression in squamous cell carcinoma and adenocarcinoma of the uterine cervix. Gynecol Oncol 2004;95(3):6107.

12. Lee EJ, McClelland M, Wang Y, Long F, Choi SH, Lee JH. Distinct DNA methylation profiles between adenocarcinoma and squamous cell carcinoma of human uterine cervix. Oncol Res. 2010;18(9):401-8.

13. Dahlstrom LA, Ylitalo N, Sundstrom K, Palmgren J, Ploner A, Eloranta $\mathrm{S}$, et al. Prospective study of human papillomavirus and risk of cervical adenocarcinoma. Int J Cancer. 2010 Oct 15;127(8):1923-30.

14. Cohen Y, Singer G, Lavie O, Dong SM, Beller U, Sidransky D. The RASSF1A tumor suppressor gene is commonly inactivated in adenocarcinoma of the uterine cervix. Clin Cancer Res 2003;9(8):2981-4.
15. Chen RJ, Lin YH, Chen CA, Huang SC, Chow SN, Hsieh CY. Influence of histologic type and age on survival rates for invasive cervical carcinoma in Taiwan. Gynecol Oncol 1999; 73(2):184-90.

16. Galic V, Herzog TJ, Lewin SN, Neugut AI, Burke WM, Lu YS, et al. Prognostic significance of adenocarcinoma histology in women with cervical cancer. Gynecol Oncol 2012; 125(2):287-91.

17. Katanyoo K, Sanguanrungsirikul S, Manusirivithaya S. Comparison of treatment outcomes between squamous cell carcinoma and adenocarcinoma in locally advanced cervical cancer. Gynecol Oncol 2012;125(2):292-6.

18. Nag S, Chao C, Erickson B, Fowler J, Gupta N, Martinez A, et al. The American Brachytherapy Society recommendations for low-dose-rate brachytherapy for carcinoma of the cervix. Int $\mathrm{J}$ Radiat Oncol Biol Phys 2002;52(1):33-48.

19. Nag S, Erickson B, Thomadsen B, Orton C, Demanes JD, Petereit D. The American Brachytherapy Society recommendations for high-dose-rate brachytherapy for carcinoma of the cervix. Int $\mathrm{J}$ Radiat Oncol Biol Phys 2000;48(1):201-11. 
20. Walji N, Chue AL, Yap C, Rogers LJ, ElModir A, Chan KK, et al. Is there a role for adjuvant hysterectomy after suboptimal concurrent chemoradiation in cervical carcinoma? Clin Oncol (R Coll Radiol) 2010;22(2):140-6. 